infection), in dermatomyositis, and in scleroderma. In cases of rheumatoid arthritis treated with ACTH or cortisone, C-reactive protein rapidly disappeared. It was suggested that the test might be profitably used in assessing the response to other therapeutic agents with possible anti-rheumatic activity.

Radiotherapy in Ankylosing Spondylitis, by Dr. J. L. Potter.

An investigation of the clinical, haematological, and biochemical effects of radiotherapy in ten cases of ankylosing spondylitis and one case of spinal osteo-arthritis were described, and the results reported. It was suggested that the early clinical improvement observed may be mediated partly by an increased adreno-cortical activity, and further experiments to confirm this hypothesis were discussed.

The Sterno-Manubrial Joint in Rheumatoid Arthritis and Ankylosing Spondylitis, by Dr. Bruce Cruickshank.

The histological changes which occur in the manubrio-sternal joint and in symphysis pubis in ankylosing spondylitis were discussed, illustrated, and correlated with the radiological appearances. It was suggested that the pathological process in these cartilaginous joints is analogous to that seen in synovial joints in the disease.

Clinical and Metabolic Effects of Glutathione in Rheumatoid Arthritis, by Dr. A. P. Meiklejohn.

The clinical effect obtained by the intravenous administration of glutathione to a case of rheumatoid arthritis was described, but it was reported that attempts to repeat this effect had met with failure. The theoretical implications were discussed with special reference to the utilization of vitamin $\mathrm{C}$ in rheumatoid arthritis.

A Method of Demonstrating the Union of Antibody and Antigen in Tissues, by Dr. Alan G. S. Hill.

The use of fluorescein-tagged antibody as a specific cytochemical reagent for the detection of antigenic material in tissues was described. Illustrations were shown of results obtained in a study of the localization of an injected bacterial polysaccharide in animal tissues. The possibility of applying the method to a study of the rheumatic diseases was discussed.

\title{
CLINICAL MEETING
}

The Heberden Society met at the Ciba Foundation, 41 Portland Place, London, W.1, on July 27, 1951. The President, Professor Sir Henry Cohen, was in the Chair.

Dr. W. H. Bradley gave a paper on The Antagonism between Jaundice and Rheumatism. He said that intermittent and spontaneous recovery was an unpredictable feature of the chronic rheumatic diseases, but that there was a persistent claim that certain acute infections, pregnancy, and jaundice antagonized rheumatism. The first important contribution to this subject had been made in 1933 by Hench, who by 1940 had collected 72 cases in which jaundice appeared to ameliorate rheumatism and wrote that:

Within the bodies of patients who have even the severest arthritis powerful forces lie dormant which merely await proper stimulation.

Light on the subject had since come from:

(a) experimental induction of hyperbilirubinuria.

(b) jaundice incidental to therapy with synthetic analgesic drugs: Lactophen, Atophan, Nirvanol, and, in a special class, gold. 
The transmission of hepatitis due to biological agents had first been attempted in 1943 as part of the work of the Medical Research Council Jaundice Committee, and transmission of epidemic hepatitis and homologous serum jaundice to rheumatoids had been effected. The results supported Hench's opinion that:

Somehow hepatitis provides a stimulant, following which a rapid and dramatic even if temporary recovery from arthritis may occur.

The following points regarding the ameliorative effect were noted by the Committee:

(i) it occurred early in the pre-icteric phase of hepatitis when erythematous rashes might also occur in hepatitis, or in Lactophen jaundice, or with Atophan;

(ii) it appeared to be independent of the presence of jaundice or of the serum bilirubin level, anorexia or starvation, fever, decubitus, and absence of bile from the gut;

(iii) it was not merely the result of a depression of the pain threshold;

(iv) it occurred only in the presence of rheumatic activity, and quiescent and burntout rheumatoids did not benefit;

(v) an impression was obtained that return of rheumatic activity depended upon restoration of normal hepatic functions.

The effect of hepatitis closely resembled the effect of cortisone, and since there was a possibility that both these "cures" were brought about in the same way, a search for common factors should be instructive. A comparison of total circulating protein in hepatitis and in rheumatoid arthritis appeared to give a lead, and it seemed that the collagenolytic effect of cortisone and its influence on antibody productions was worth exploration.

For the time being, both cortisone and hepatitis should be regarded as no more than research tools-both dangerous-and special precautions were necessary if the hepatitis virus was used in clinical investigation.

Drs E. G. L. Bywaters and A. St. J. Dixon presented a paper on Intra-Articular Cortisone in Rheumatoid Arthritis. The patients selected for this study had symmetrical effusions in the knees due to rheumatoid arthritis. One knee received injections of intraarticular cortisone acetate, and the other knee received blank injections. The injections were made after complete aspiration of the joint fluid. Comparison between the fluids found in both knees 24 and 48 hrs later showed that cortisone acetate reduced the volume of the fluid formed in the treated knee and increased its viscosity. The increase of viscosity was shown to be due to an increased polymerization of hyaluronic acid.

Serial injections into the knee joint at 4-day intervals, using increasing doses of cortisone acetate (with the contra-lateral knee as a control), showed that it was possible, with adequate dose, almost completely to inhibit the re-formation of joint fluid; and that the little which was re-formed was of high viscosity. A further series of injections was given (again treating one knee and using the other as a control). After a control period the patient was given a short course of systemic salicylates. No additive effect on salicylates and cortisone could be demonstrated.

On one patient the least intra-articular dose which could be shown to produce an alteration in joint fluid was $20 \mathrm{mg}$., and the greatest dose which could be-injected into the knee joint without systemic effects was $160 \mathrm{mg}$.

Other results of the intra-articular administration of cortisone were:

(i) decrease in the total white-cell count with relative increase in lymphocytes in the fluid;

(ii) no change in the total and differential protein content in the fluid;

(iii) no change in the fluid glucosamine or glucose;

(iv) alteration in the type of clot formed with acetic acid when added to the joint fluid (indicating an increase of polymerization of hyaluronic acid). 
However, in vitro, the addition of cortisone acetate suspension to joint fluid showed that the former is precipitated rapidly as fibrin-covered aggregates. This might explain how it was that the quantitative effect of any particular dose of intra-articular cortisone could not be forecast with certainty.

Dr. Fred Wrigley gave a paper on Experimental Arthritis: Its Production and Measurement. Work on the production of joint swelling in animals had first been started by making use of the pleuropneumonia-like organism. Early experiences showed that abscesses were frequently produced in the animals at the same time as the joint swelling. Animal passage, sub-culture, and freeze-drying, had been employed, and a preparation finally obtained which, on injection, caused joint swelling without abscess formation. A study of this condition had shown that it was capable of spontaneous resolution, and therefore the animals were not used for the screening of drugs.

"Formalin arthritis" had been produced in a large number of animals, and although peri-articular swelling was apparent, there was no dramatic alteration in the condition, nor any prevention of the development of the condition when ACTH, cortisone, DOCA, and DOCA and ascorbic acid were administered. These results were controlled by:

(a) uninjected animals;

(b) formalin-injected animals without further medication.

Throughout the work it was obvious that a method of measuring joint swelling must be devised, and a method was ultimately adopted in which the animal's limb was exposed to light over photographic paper and a silhouette obtained. The shapes from different positions were weighed and a figure obtained representing the volume of the limb. These very readily allowed a group comparison, and showed up changes in limb volume. The method was checked by comparison with an inanimate object and shown to be accurate to within plus or minus 6 per cent.

Dr. C. J. M. Clark gave a paper entitled Observations on Rose's Agglutination Test in Rheumatoid Arthritis. The development of Rose's sheep-cell agglutination test was described, and the results in a series of cases discussed. In the technique used the heterophil agglutinin was not absorbed and a 2-hr reading was used. A titre of 1 in 32 or over was considered positive. There were no positive reactions among 42 patients with ankylosing spondylitis. Two out of twenty patients with miscellaneous arthritic lesions had positive titres. Both these appeared clinically to be cases of palindromic rheumatism but their follow-up was short.

Out of 100 patients with rheumatoid arthritis, 33 had positive reactions and an analysis was made of these cases. Graphs showed that there was no direct relation between the titre and any of the following factors:

haemoglobin
packed-cell volume
erythrocyte sedimentation rate
serum albumin
serum globulin

serum colloidal gold thymol flocculation thymol turbidity zinc sulphate turbidity ammonium sulphate turbidity.

In addition, no correlation with changes in plasma fibrinogen, splenic, or lymph-node enlargement, nodule formation, or gold therapy could be established, but it did appear that only low titres were found in cases with a long history of arthritis who had a positive agglutination test.

No obvious relationship to the activity of disease could be established, but preliminary results did suggest that ACTH and cortisone reduced the titre of the positive reactions in some cases.

Dr. D. LoNG gave a paper on Cortisone and Allergy which is to be published in full in a later issue of the Annals. 